\title{
Ising Spin Glass Under Continuous-Distribution Random Magnetic Fields: Tricritical Points and Instability Lines
}

\author{
Nuno Crokidakit* and Fernando D. Nobret \\ Centro Brasileiro de Pesquisas Físicas \\ Rua Xavier Sigaud 150 \\ 22290-180 Rio de Janeiro - RJ Brazil
}

(Dated: October 29, 2018)

The effects of random magnetic fields are considered in an Ising spin-glass model defined in the limit of infinite-range interactions. The probability distribution for the random magnetic fields is a double Gaussian, which consists of two Gaussian distributions centered respectively, at $+H_{0}$ and $-H_{0}$, presenting the same width $\sigma$. It is argued that such a distribution is more appropriate for a theoretical description of real systems than its simpler particular two well-known limits, namely the single Gaussian distribution $\left(\sigma \gg H_{0}\right)$, and the bimodal one $(\sigma=0)$. The model is investigated by means of the replica method, and phase diagrams are obtained within the replica-symmetric solution. Critical frontiers exhibiting tricritical points occur for different values of $\sigma$, with the possibility of two tricritical points along the same critical frontier. To our knowledge, it is the first time that such a behavior is verified for a spin-glass model in the presence of a continuous-distribution random field, which represents a typical situation of a real system. The stability of the replicasymmetric solution is analyzed, and the usual Almeida-Thouless instability is verified for low temperatures. It is verified that, the higher-temperature tricritical point always appears in the region of stability of the replica-symmetric solution; a condition involving the parameters $H_{0}$ and $\sigma$, for the occurrence of this tricritical point only, is obtained analytically. Some of our results are discussed in view of experimental measurements available in the literature.

Keywords: Spin Glasses, Random-Field Systems, Replica Method, Almeida-Thouless Instability. 
PACS numbers: $05.50+\mathrm{q}, 64.60 .-\mathrm{i}, 75.10 . \mathrm{Nr}, 75.50 . \mathrm{Lk}$

\section{INTRODUCTION}

Spin-glass systems [1, 2, 3, 4, 5] continue to challenge many researchers in the area of magnetism. ¿From the theoretical point of view, its simplest version defined in terms of Ising spin variables, the so-called Ising spin glass (ISG), represents one of the most fascinating problems in the physics of disordered magnets. The ISG mean-field solution, based on the infinite-range-interaction model, as proposed by Sherrington-Kirkpatrick (SK) [6], presents a quite nontrivial behavior. The correct low-temperature solution of the SK model is defined in terms of a continuous order-parameter function [7] (i.e., an infinite number of order parameters) associated with many low-energy states, a procedure which is usually denominated as replica-symmetry breaking (RSB). Furthermore, a transition in the presence of an external magnetic field, known as the Almeida-Thouless (AT) line [8], is found in the solution of the SK model: such a line separates a low-temperature region, characterized by RSB, from a high-temperature one, where a simple one-parameter solution, denominated as replica-symmetric $(\mathrm{RS})$ solution, is stable. The validity of the results of the SK model for the description of more realistic systems, characterized by short-rangeinteractions, represents a very polemic question [5]. Recent numerical simulations claim the absence of an AT line in the three-dimensional short-range ISG [9], as well as along the non-mean-field region of a one-dimensional ISG characterized by long-range interactions [10]. However, these results, obtained with rather small lattice-size simulations, do not rule out the possibility of a crossover to a different scenario at much larger lattice sizes, or also for smaller fields (and/or temperatures). One candidate for alternative theory to the SK model is the droplet model [11], based on domain-wall renormalization-group arguments for spin glasses [12, 13]. According to the droplet model, the low-temperature phase of any finite-dimensional short-range spin glass should be described in terms of

\footnotetext{
*E-mail address: nuno@cbpf.br

${ }^{\dagger}$ Corresponding author: E-mail address: fdnobre@cbpf.br
} 
a single thermodynamic state (together, of course, with its corresponding time-reversed counterpart), i.e., essentially a RS-type of solution. Many important features of the ISG still deserve an appropriate understanding within the droplet-model scenario, and in particular, the validity of this model becomes questionable for increasing dimensionalities, where one expects the existence of a finite upper critical dimension, above which the mean-field picture should prevail.

Some diluted antiferromagnets, like $\mathrm{Fe}_{\mathrm{x}} \mathrm{Zn}_{1-\mathrm{x}} \mathrm{F}_{2}, \mathrm{Fe}_{\mathrm{x}} \mathrm{Mg}_{1-\mathrm{x}} \mathrm{Cl}_{2}$ and $\mathrm{Mn}_{\mathrm{x}} \mathrm{Zn}_{1-\mathrm{x}} \mathrm{F}_{2}$, have been the object of extensive experimental research, due to their intriguing properties [14]. These systems are able to exhibit, within certain concentration ranges, randomfield, spin-glass or both behaviors, and in particular, the compounds $\mathrm{Fe}_{\mathrm{x}} \mathrm{Zn}_{1-\mathrm{x}} \mathrm{F}_{2}$ and $\mathrm{Fe}_{\mathrm{x}} \mathrm{Mg}_{1-\mathrm{x}} \mathrm{Cl}_{2}$ are characterized by large crystal-field anisotropies, in such a way that they may be reasonably well-described in terms of Ising variables. Therefore, they are usually considered as good physical realizations of the random-field Ising model (RFIM), or also of an ISG. For the $\mathrm{Fe}_{\mathrm{x}} \mathrm{Zn}_{1-\mathrm{x}} \mathrm{F}_{2}$, one gets a RFIM-like behavior for $\mathrm{x}>0.42$, an ISG for $\mathrm{x} \sim 0.25$, whereas for intermediate concentrations $(0.25<\mathrm{x}<0.42)$ one may observe both behaviors depending on the magnitude of the applied external magnetic field [RFIM (ISG) for small (large) magnetic fields], with a crossover between them [15, 16, 17]. In what concerns $\mathrm{Fe}_{\mathrm{x}} \mathrm{Mg}_{1-\mathrm{x}} \mathrm{Cl}_{2}$, one gets an ISG-like behavior for $\mathrm{x}<0.55$, whereas for $0.7<$ $\mathrm{x}<1.0$ one has a typical RFIM with a first-order transition turning into a continuous one due to a change in the random fields [14, 18, 19]. Even though a lot of experimental data is available for these systems, they still deserve an appropriate understanding, with only a few theoretical models proposed for that purpose [20, 21, 22, 23, 24, 25, 26, 27]. Within the numerical-simulation technique, one has tried to take into account the basic microscopic ingredients of such systems [20, 21, 22, 23], whereas at the mean-field level, a joint study of both ISG and RFIM models has been shown to be a very promising approach [24, 25, 26, 27].

In the present work we investigate the effects of random magnetic fields, following a continuous probability distribution, in an ISG model. The model is considered in the limit of infinite-range interactions, and the probability distribution for the random magnetic fields is a double Gaussian, which consists of a sum of two independent Gaussian distributions. Such a distribution interpolates between the bimodal and the simple Gaus- 
sian distributions, which are known to present distinct low-temperature critical behavior, within the mean-field limit [24, 25, 26, 27]. It is argued that this distribution is more appropriate for a theoretical description of diluted antiferromagnets than the bimodal and Gaussian distributions. In particular, for given ranges of parameters, this distribution presents two peaks, and satisfies the requirement of effective random fields varying in both sign and magnitude, which comes out naturally in the identification of the RFIM with diluted antiferromagnets in the presence of a uniform field [28, 29]; this condition is not fulfilled by simple discrete probability distributions, e.g., the bimodal one, which is certainly very convenient from the theoretical point of view. Recently, the use a doubleGaussian distribution in the RFIM [30] yielded interesting results, leading to a candidate model to describe the change of a first-order transition into a continuous one that occurs in $\mathrm{Fe}_{\mathrm{x}} \mathrm{Mg}_{1-\mathrm{x}} \mathrm{Cl}_{2}$ [14, 18, 19]. The use of this distribution in the study of the present model should be relevant for $\mathrm{Fe}_{\mathrm{x}} \mathrm{Mg}_{1-\mathrm{x}} \mathrm{Cl}_{2}$ with concentrations $x<0.55$, where the ISG behavior shows up. In the next section we study the SK model in the presence of the above-mentioned random magnetic fields; a rich critical behavior is presented, and in particular, one finds a critical frontier that may present one, or even two, tricritical points. The instabilities of the RS solution are also investigated, and AT lines presenting an inflection point, in concordance with those measured in some diluted antiferromagnets, are obtained. Finally, in section 4 we present our conclusions.

\section{THE ISING SPIN GLASS IN THE PRESENCE OF A RANDOM-FIELD}

The infinite-range-interaction Ising spin-glass model, in the presence of an external random magnetic field, may be defined in terms of the Hamiltonian

$$
\mathcal{H}=-\sum_{(i, j)} J_{i j} S_{i} S_{j}-\sum_{i} H_{i} S_{i}
$$

where the sum $\sum_{(i, j)}$ applies to all distinct pairs of spins $S_{i}= \pm 1(i=1,2, \ldots, N)$. The interactions $\left\{J_{i j}\right\}$ and the fields $\left\{H_{i}\right\}$ follow independent probability distributions, 

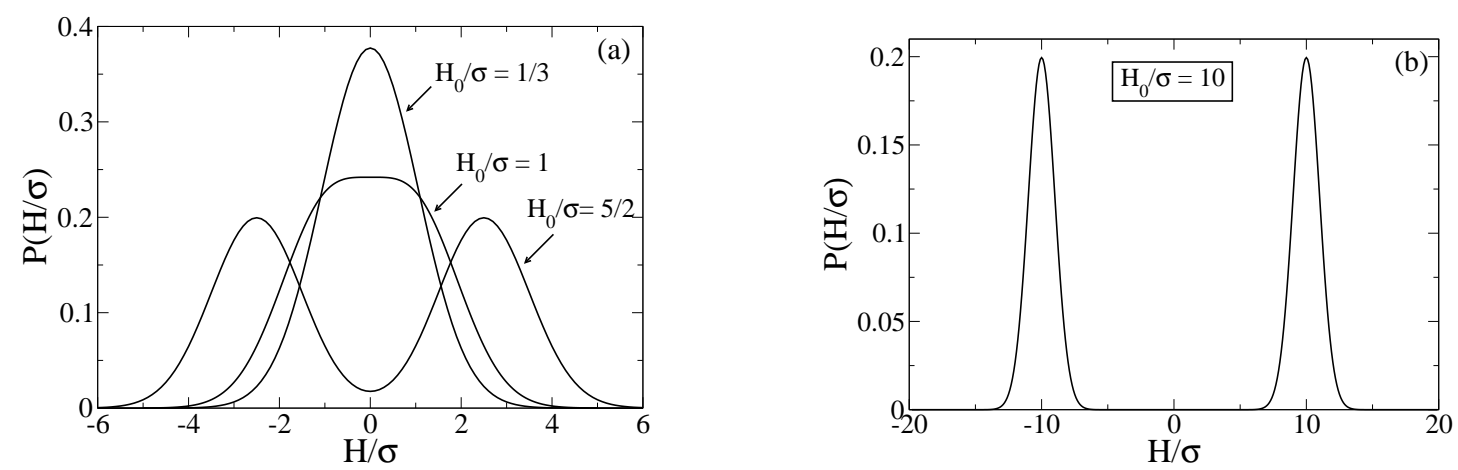

FIG. 1: The probability distribution of Eq. (3) (the random fields are scaled in units of $\sigma$ ) for typical values of the ratio $H_{0} / \sigma$ : (a) $\left(H_{0} / \sigma\right)=1 / 3,1,5 / 2$; (b) $\left(H_{0} / \sigma\right)=10$.

$$
\begin{gathered}
P\left(J_{i j}\right)=\left(\frac{N}{2 \pi J^{2}}\right)^{1 / 2} \exp \left[-\frac{N}{2 J^{2}}\left(J_{i j}-\frac{J_{0}}{N}\right)^{2}\right], \\
P\left(H_{i}\right)=\frac{1}{2}\left(\frac{1}{2 \pi \sigma^{2}}\right)^{1 / 2}\left\{\exp \left[-\frac{\left(H_{i}-H_{0}\right)^{2}}{2 \sigma^{2}}\right]+\exp \left[-\frac{\left(H_{i}+H_{0}\right)^{2}}{2 \sigma^{2}}\right]\right\} .
\end{gathered}
$$

The probability distribution for the fields $\left\{H_{i}\right\}$ is a double Gaussian and depends on two parameters, $H_{0}$ and $\sigma$, modifying its form according to the ratio $H_{0} / \sigma$, as exhibited in Fig. 1. Such a distribution is double-peaked for $\left(H_{0} / \sigma\right)>1$, presents a single peak for $\left(H_{0} / \sigma\right)<1$, changing its concavity at the origin when $\left(H_{0} / \sigma\right)=1$. Besides that, in the limit $\sigma \rightarrow 0$, one recovers a bimodal probability distribution. It is important to notice that its kurtosis, $\kappa=<H_{i}^{4}>_{H} /\left[3\left(<H_{i}^{2}>_{H}\right)^{2}\right]\left[\right.$ where $<>_{H}$ denotes an average over $\left.P\left(H_{i}\right)\right]$, varies from $\kappa=1 / 3$ (bimodal limit) to $\kappa=1$ (Gaussian limit), approaching unit only in the limit $\left(H_{0} / \sigma\right) \rightarrow 0$, in which case one gets a perfect Gaussian distribution. For finite values of $H_{0} / \sigma$ one gets $1 / 3<\kappa<1$, and in particular, for the cases exhibited in Fig. 1 one has that $\kappa \approx 0.99\left[\left(H_{0} / \sigma\right)=1 / 3\right], \kappa \approx 0.83\left[\left(H_{0} / \sigma\right)=1\right], \kappa \approx 0.50\left[\left(H_{0} / \sigma\right)=5 / 2\right]$, and $\kappa \approx 0.35\left[\left(H_{0} / \sigma\right)=10\right]$.

The free energy of a given disorder realization, $F\left(\left\{J_{i j}, H_{i}\right\}\right)$, depends on two random variables, in such a way that the average over the disorder [ ] $]_{J, H}$ may be written in terms of independent integrals, 


$$
\left[F\left(\left\{J_{i j}, H_{i}\right\}\right)\right]_{J, H}=\int \prod_{(i, j)}\left[d J_{i j} P\left(J_{i j}\right)\right] \prod_{i}\left[d H_{i} P\left(H_{i}\right)\right] F\left(\left\{J_{i j}, H_{i}\right\}\right)
$$

Now, one can make use of the replica method [1, 2, 3, 4] in order to obtain the free energy per spin,

$$
-\beta f=\lim _{N \rightarrow \infty} \frac{1}{N}\left[\ln Z\left(\left\{J_{i j}, H_{i}\right\}\right)\right]_{J, H}=\lim _{N \rightarrow \infty} \lim _{n \rightarrow 0} \frac{1}{N n}\left(\left[Z^{n}\right]_{J, H}-1\right)
$$

where $Z^{n}$ represents the partition function of the replicated system and $\beta=1 /(k T)$. Standart calculations lead to

$$
\beta f=-\frac{(\beta J)^{2}}{4}-\frac{(\beta \sigma)^{2}}{2}+\lim _{n \rightarrow 0} \frac{1}{n} \min g\left(m^{\alpha}, q^{\alpha \beta}\right)
$$

where

$$
\begin{aligned}
g\left(m^{\alpha}, q^{\alpha \beta}\right) & =\frac{\beta J_{0}}{2} \sum_{\alpha}\left(m^{\alpha}\right)^{2}+\frac{(\beta J)^{2}}{2} \sum_{(\alpha \beta)}\left(q^{\alpha \beta}\right)^{2}-\frac{1}{2} \ln \operatorname{Tr}_{\alpha} \exp \left(\mathcal{H}_{\text {eff }}^{+}\right)-\frac{1}{2} \ln \operatorname{Tr}_{\alpha} \exp \left(\mathcal{H}_{\text {eff }}^{-}\right) \\
\mathcal{H}_{\text {eff }}^{ \pm} & =\beta J_{0} \sum_{\alpha} m^{\alpha} S^{\alpha}+(\beta \sigma)^{2} \sum_{(\alpha \beta)} S^{\alpha} S^{\beta}+(\beta J)^{2} \sum_{(\alpha \beta)} q^{\alpha \beta} S^{\alpha} S^{\beta} \pm \beta H_{0} \sum_{\alpha} S^{\alpha} .
\end{aligned}
$$

In the equations above, the index $\alpha(\alpha=1,2, \ldots, n)$ is a replica label, $\operatorname{Tr}_{\alpha}$ represents a trace over the spin variables of each replica, and $\sum_{(\alpha \beta)}$ denote sums over distinct pairs of replicas.

The extrema of the functional $g\left(m^{\alpha}, q^{\alpha \beta}\right)$ give us the equilibrium equations

$$
\begin{aligned}
m^{\alpha} & =\frac{1}{2}<S^{\alpha}>_{+}+\frac{1}{2}<S^{\alpha}>_{-}, \\
q^{\alpha \beta} & =\frac{1}{2}<S^{\alpha \beta}>_{+}+\frac{1}{2}<S^{\alpha \beta}>_{-} \quad(\alpha \neq \beta),
\end{aligned}
$$


where $<>_{ \pm}$indicate thermal averages with respect to the "effective Hamiltonians" $\mathcal{H}_{\text {eff }}^{ \pm}$ in Eq. (8).

Assuming the RS ansatz [1, 2, 3, 4] , i.e., $m^{\alpha}=m(\forall \alpha)$ and $q^{\alpha \beta}=q[\forall(\alpha \beta)]$, Eqs. (66)(10) yield

$$
\begin{aligned}
\beta f= & -\frac{(\beta J)^{2}}{4}(1-q)^{2}+\frac{\beta J_{0}}{2} m^{2}-\frac{1}{2} \frac{1}{\sqrt{2 \pi}} \int_{\infty}^{\infty} d z e^{-z^{2} / 2} \ln \left(2 \cosh \xi^{+}\right) \\
& -\frac{1}{2} \frac{1}{\sqrt{2 \pi}} \int_{\infty}^{\infty} d z e^{-z^{2} / 2} \ln \left(2 \cosh \xi^{-}\right), \\
m= & \frac{1}{2} \frac{1}{\sqrt{2 \pi}} \int_{-\infty}^{+\infty} d z e^{-z^{2} / 2} \tanh \xi^{+}+\frac{1}{2} \frac{1}{\sqrt{2 \pi}} \int_{-\infty}^{+\infty} d z e^{-z^{2} / 2} \tanh \xi^{-}, \\
q= & \frac{1}{2} \frac{1}{\sqrt{2 \pi}} \int_{-\infty}^{+\infty} d z e^{-z^{2} / 2} \tanh ^{2} \xi^{+}+\frac{1}{2} \frac{1}{\sqrt{2 \pi}} \int_{-\infty}^{+\infty} d z e^{-z^{2} / 2} \tanh ^{2} \xi^{-},
\end{aligned}
$$

where

$$
\begin{gathered}
\xi^{ \pm}=\beta\left\{J_{0} m+J G z \pm H_{0}\right\} \\
G=\left[q+\left(\frac{\sigma}{J}\right)^{2}\right]^{1 / 2}
\end{gathered}
$$

Although the spin-glass order parameter [Eq. (13)] is always induced by the random field, it may still contribute to a nontrivial behavior. The RS solution is known to lead to an instability at low temperatures, usually associated to this parameter, occurring below the AT [8] line,

$$
\left(\frac{k T}{J}\right)^{2}=\frac{1}{2} \frac{1}{\sqrt{2 \pi}} \int_{-\infty}^{+\infty} d z e^{-z^{2} / 2} \operatorname{sech}^{4} \xi^{+}+\frac{1}{2} \frac{1}{\sqrt{2 \pi}} \int_{-\infty}^{+\infty} d z e^{-z^{2} / 2} \operatorname{sech}^{4} \xi^{-}
$$

Let us now present the phase diagrams of this model. Since the random field induces the parameter $q$, there is no spontaneous spin-glass order, like the one found in the SK model. However, there is a phase transition related to the magnetization $m$, in such a way 
that two phases are possible within the RS solution, namely, the Ferromagnetic $(m \neq 0$, $q \neq 0)$ and the Independent $(m=0, q \neq 0)$ ones. The critical frontier separating these two phases is obtained by solving the equilibrium conditions, Eqs. (12) and (13), whereas in the case of first-order phase transitions, the free energy per spin, Eq. (11), will be analyzed. Expanding the magnetization [Eq. (12)] in power series,

$$
m=A_{1}(q) m+A_{3}(q) m^{3}+A_{5}(q) m^{5}+O\left(m^{7}\right)
$$

where

$$
\begin{aligned}
& A_{1}(q)=\beta J_{0}\left\{1-\rho_{1}(q)\right\} \\
& A_{3}(q)=-\frac{\left(\beta J_{0}\right)^{3}}{3}\left\{1-4 \rho_{1}(q)+3 \rho_{2}(q)\right\} \\
& A_{5}(q)=\frac{\left(\beta J_{0}\right)^{5}}{15}\left\{2-17 \rho_{1}(q)+30 \rho_{2}(q)-15 \rho_{3}(q)\right\}
\end{aligned}
$$

and

$$
\rho_{k}(q)=\frac{1}{\sqrt{2 \pi}} \int_{-\infty}^{+\infty} d z e^{-z^{2} / 2} \tanh ^{2 k} \beta J\left\{G z+\frac{H_{0}}{J}\right\} .
$$

The coefficients in Eqs. (18)-(20) depend on $q$, which in its turn depends on $m$ through Eq. (13). In order to eliminate this dependence, we expand Eq. (13) in powers of $m$,

$$
q=q_{0}+\left(\beta J_{0}\right)^{2} \frac{\Gamma}{1-(\beta J)^{2} \Gamma} m^{2}+O\left(m^{4}\right)
$$

with

$$
\Gamma=1-4 \rho_{1}\left(q_{0}\right)+3 \rho_{2}\left(q_{0}\right)
$$

where $q_{0}$ corresponds to the solution of Eq. (13) for $m=0$. Substituting Eq. (22) in the expansion of Eq. (17), one obtains the $m$-independent coefficients in the power expansion 
of the magnetization; in terms of the lowest-order coefficients, one gets,

$$
\begin{aligned}
m & =A_{1}^{\prime} m+A_{3}^{\prime} m^{3}+O\left(m^{5}\right), \\
A_{1}^{\prime} & =A_{1}\left(q_{0}\right), \\
A_{3}^{\prime} & =-\frac{\left(\beta J_{0}\right)^{3}}{3}\left[\frac{1+2(\beta J)^{2} \Gamma}{1-(\beta J)^{2} \Gamma}\right] \Gamma .
\end{aligned}
$$

The associated critical frontier is determined through the standard procedure, taking into account the spin-glass order parameter [Eq. (13)], as well. For continuous transitions, $A_{1}^{\prime}=1$, with $A_{3}^{\prime}<0$, in such a way that one has to solve numerically the equation $A_{1}^{\prime}=1$, together with Eq. (13) considering $m=0$. If $A_{3}^{\prime}>0$, one may have first-order phase transitions, characterized by a discontinuity in the magnetization; in this case, the critical frontier is found through a Maxwell construction, i.e., by equating the free energies of the two phases, which should be solved numerically together with Eqs. (12) and (13) for each side of the critical line. When both types of phase transitions are present, the continuous and first-order critical frontiers meet at a tricritical point that defines the limit of validity of the series expansion. The location of such a point is determined by solving numerically equations $A_{1}^{\prime}=1, A_{3}^{\prime}=0$, and Eq. (13) with $m=0$ [provided that the coefficient of the next-order term in the expansion of Eq. (24) is negative, i.e., $\left.A_{5}^{\prime}<0\right]$.

Considering the above-mentioned phases, the AT instability of Eq. (16) splits each of them in two phases, in such a way that the phase diagram of this model may present four phases, that are usually classified as [24, 25, 26]: (i) Paramagnetic (P) $(m=0$; stability of the RS solution); (ii) Spin-Glass (SG) ( $m=0$; instability of the RS solution); (iii) Ferromagnetic $(\mathrm{F})\left(m \neq 0\right.$; stability of the RS solution); (iv) Mixed Ferromagnetic $\left(\mathrm{F}^{\prime}\right)$ ( $m \neq 0$; instability of the RS solution).

Even though in most cases the AT line is computed numerically, for large values of $J_{0}$ [i.e., $J_{0}>>J$ and $J_{0}>>H_{0}$ ] and low temperatures, one gets the following analytic asymptotic behavior,

$$
\frac{k T}{J} \cong \frac{2}{3} \frac{1}{\sqrt{2 \pi}} \frac{1}{G}\left\{\exp \left[-\frac{\left(J_{0}+H_{0}\right)^{2}}{2 J^{2} G^{2}}\right]+\exp \left[-\frac{\left(J_{0}-H_{0}\right)^{2}}{2 J^{2} G^{2}}\right]\right\} .
$$



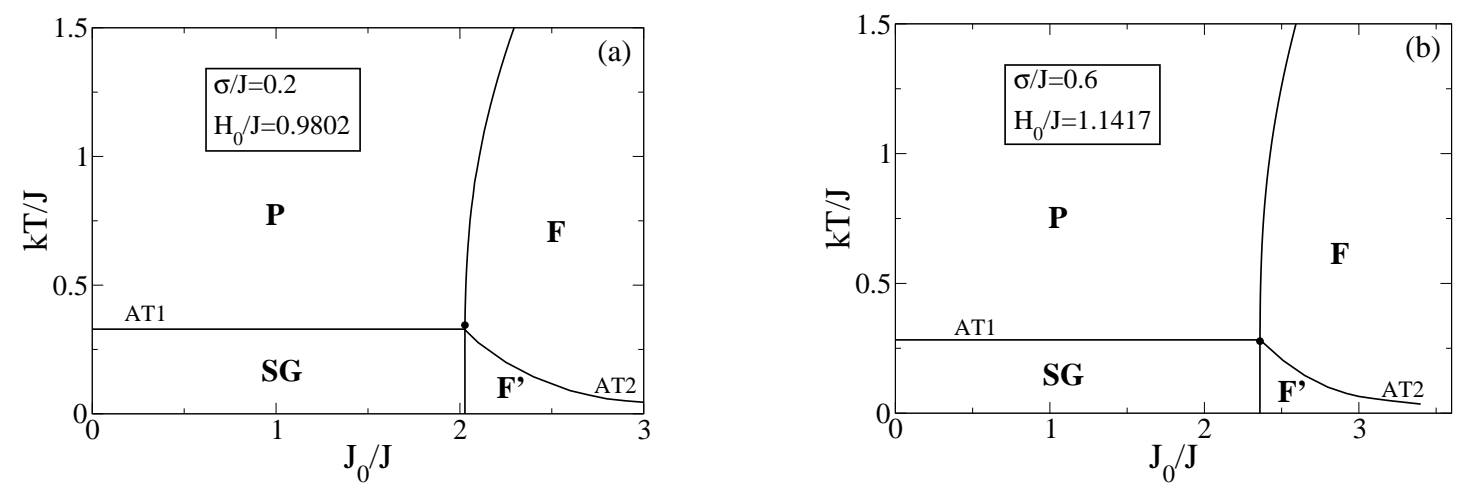

FIG. 2: Phase diagrams of the infinite-range-interaction ISG in the presence of a double-Gaussian random field; the phases are labelled according to the definitions in the text. AT1 and AT2 denote AT lines, and all variables are scaled in units of $J$. Two typical examples $[($ a) $(\sigma / J)=0.2$; (b) $(\sigma / J)=0.6]$ are exhibited, for which there are single points (represented by black dots) characterized by $A_{1}^{\prime}=1$ and $A_{3}^{\prime}=0$, defining the corresponding threshold values $H_{0}^{(1)}(\sigma)$.

For the particular case $\sigma=0$, i.e., the bimodal probability distribution for the fields [25], it was verified that the phase diagrams of the model change qualitatively and quantitatively for incresing values of $H_{0}$. Herein, we show that the phase diagrams of the present model change according to the parameters of the distribution of random fields [Eq. (3)], which may modify drastically the critical line separating the regions with $m=0$ and $m \neq 0$, defined by the coefficients in Eq. (24). In particular, one finds numerically a threshold value, $H_{0}^{(1)}(\sigma)$, for which this line presents a single point characterized by $A_{1}^{\prime}=1$ and $A_{3}^{\prime}=0$; all other points of this line represent continuous phase transitions, characterized by $A_{1}^{\prime}=1$ and $A_{3}^{\prime}<0$. Typical examples of this case are exhibited in Fig. 2 , for the dimensionless ratios $(\sigma / J)=0.2$ and $(\sigma / J)=0.6$. As will be seen in the next figures, for values of $H_{0} / J$ slightly larger than $H_{0}^{(1)}(\sigma) / J$, this special point splits in two tricritical points, whereas for values of $H_{0} / J$ smaller than $H_{0}^{(1)}(\sigma) / J$, this critical frontier is completely continuous. Therefore, one may interpret the point for which $H_{0}=H_{0}^{(1)}(\sigma)$ as a collapse of two tricritical points. Such an unusual critical point is a characteristic of some infinite-range-interaction spin-glasses in the presence of random magnetic fields [25, 26], and to our knowledge, it has never been found in other magnetic models. ¿From Fig. 2, one notices that the threshold value $H_{0}^{(1)}(\sigma) / J$ increases for increasing values of 
$\sigma / J$, although the corresponding ratio $H_{0}^{(1)}(\sigma) / \sigma$ decreases. Apart from that, this peculiar critical point always occurs very close to the onset of RSB; indeed, for $(\sigma / J)=0.6$, this point essentially coincides with the union of the two AT lines (AT1 and AT2). At least for the range of ratios $\sigma / J$ investigated, this point never appeared below the AT lines, i.e., in the region of RSB. Therefore, an analysis that takes into account RSB, will not modify the location of this point in these cases.

In Fig. 3 we exhibit phase diagrams for a fixed value of $\sigma(\sigma=0.2 J)$, and increasing values of $H_{0}$. In Fig. 3(a) we show the case $\left(H_{0} / J\right)=0.5$, where one sees a phase diagram that looks like, at least qualitatively, the one of the SK model; even though the random-field distribution [cf. Eq. (3) ] is double-peaked (notice that $\left(H_{0} / \sigma\right)=2.5$ in this case), the effects of such a field are not sufficient for a qualitative change in the phase diagram of the model. As we have shown above [see Fig. 2(a)], qualitative changes only occur in the corresponding phase diagram for a ratio $\left(H_{0}^{(1)}(\sigma) / \sigma\right) \approx 5$, or higher. It is important to remark that a tricritical point occurs in the corresponding RFIM for any $\left(H_{0} / \sigma\right) \geq 1$ [30], in agreement with former general analyses [31, 32, 33]. If one associates the tricritical points that occur in the present model as reminiscents of the one in the RFIM, one notices that such effects appear attenuated in the present model due to the bond randomness, as predicted previously for short-range-interaction models [34, 35]. In Fig. 3(b) we present the phase diagram for $\left(H_{0} / J\right)=0.993$; in this case, one observes two finite-temperature tricritical points along the critical frontier that separates the regions with $m=0$ and $m \neq 0$. The higher-temperature point is located in the region where the RS approximation is stable, and so, it will not be affected by RSB effects; however, the lower-temperature tricritical point, found in the region of instability of the RS solution, may change under a RSB procedure. In Fig. 3(c) we exhibit another interesting situation of the phase diagram of this model, for which the lower-temperature tricritical point goes down to zero temperature, defining a second threshold value, $H_{0}^{(2)}(\sigma)$. This threshold value was calculated analytically, through a zero-temperature approach that follows below, for arbitrary values of $\sigma / J$. Above such a threshold, only the higher-temperature tricritical point (located in the region of stability of the RS solution) exists; this is shown in Fig. 3(d), where one considers a typical situation with $H_{0}>H_{0}^{(2)}(\sigma)$. It is important to notice that in Fig. 3(d) the two AT lines clearly do not meet at the critical frontier that separates the 

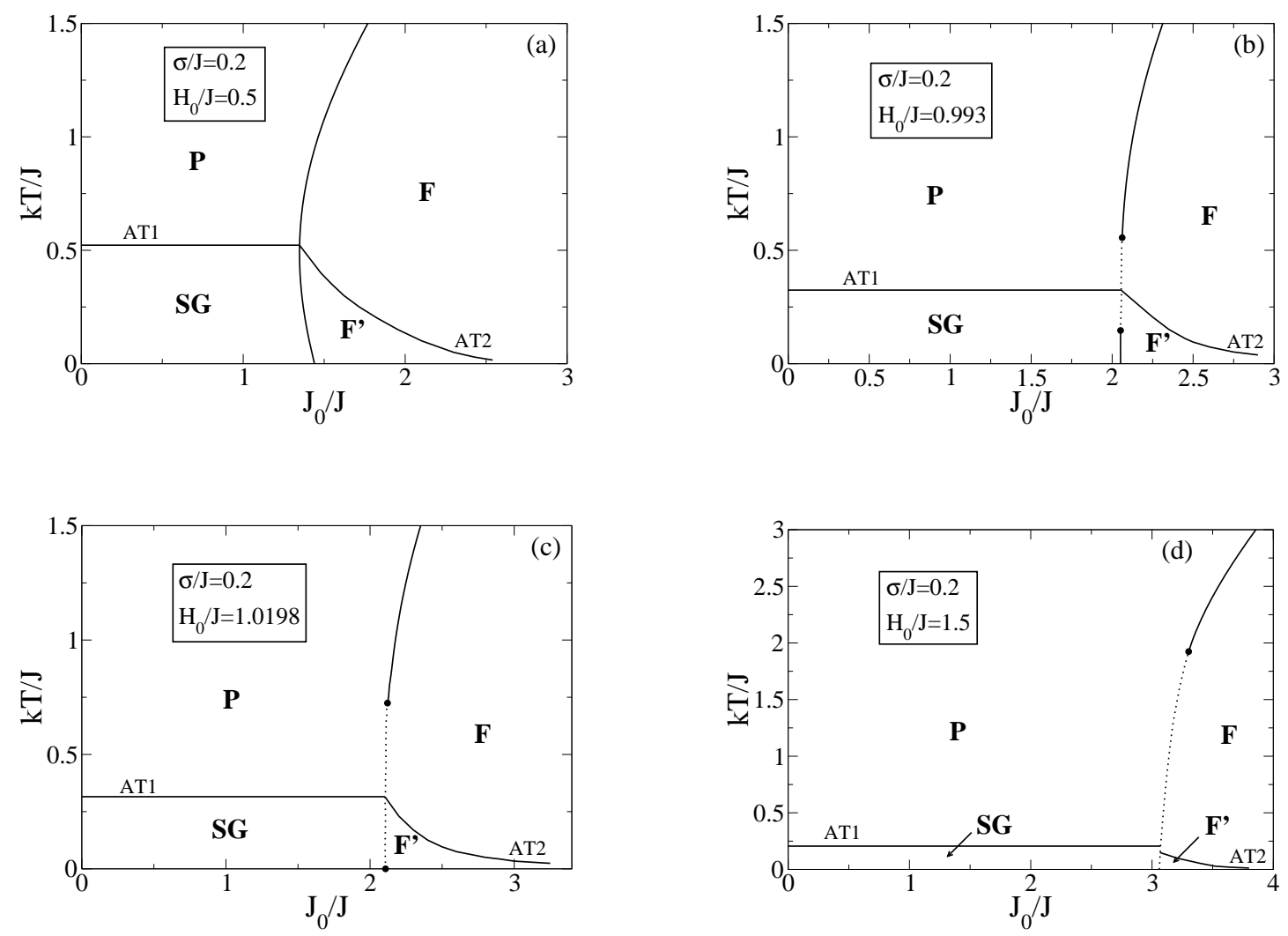

FIG. 3: Phase diagrams of the infinite-range-interaction ISG in the presence of a double-Gaussian random field with $(\sigma / J)=0.2$ and typical values of $H_{0} / J$; the phases are labelled according to the definitions in the text. AT1 and AT2 denote AT lines, and all variables are scaled in units of $J$. By increasing the value of $H_{0} / J$, the phase diagram changes both qualitatively and quantitatively and, particularly, the critical lines separating the regions with $m=0$ and $m \neq 0$ are modified; along these critical frontiers, the full (dotted) lines represent continuous (first-order) phase transitions and the black dots denote tricritical points; for the values of $H_{0} / J$ chosen, one has: (a) continuous phase transitions; (b) two tricritical points at finite temperatures; (c) the lower tricritical point at zero temperature, defining the corresponding threshold value $H_{0}^{(2)}(\sigma) ;(\mathrm{d})$ a single tricritical point at finite temperatures.

regions with $m=0$ and $m \neq 0$; such an effect is a consequence of the phase coexistence region, characteristic of first-order phase transitions, and has already been observed in the SK model with a bimodal random-field distribution [25]. The line AT1 is valid up to the right end limit of the phase coexistence region, whereas AT2 remains valid up to the 

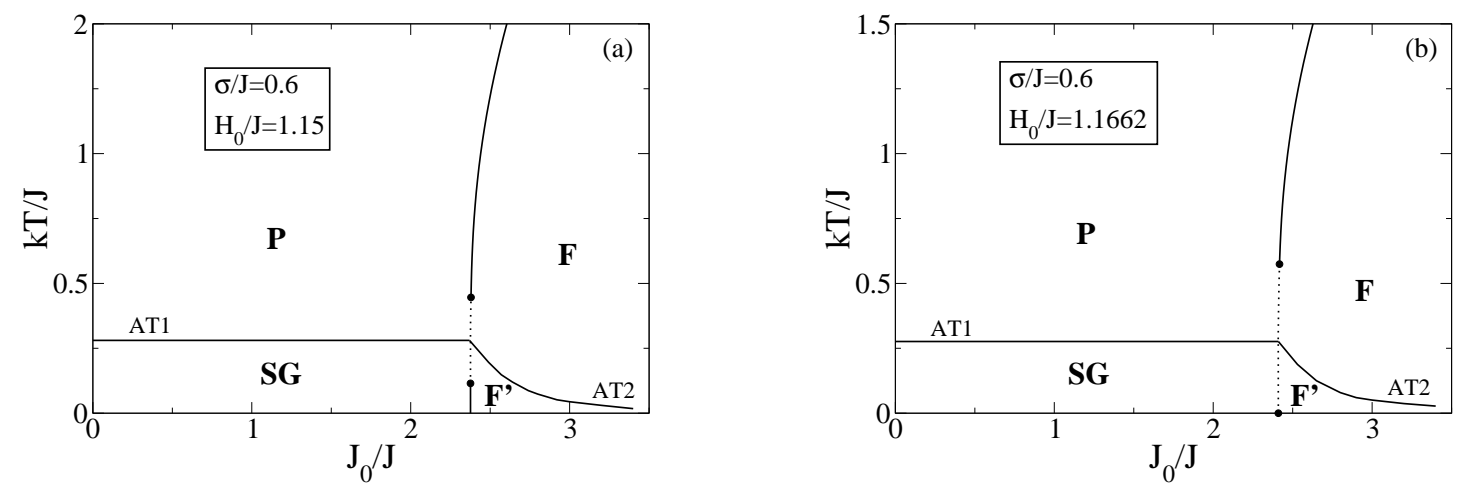

FIG. 4: Phase diagrams of the infinite-range-interaction ISG in the presence of a double-Gaussian random field with $(\sigma / J)=0.6$ and typical values of $H_{0} / J$; the phases are labelled according to the definitions in the text. AT1 and AT2 denote AT lines, and all variables are scaled in units of $J$. Along the critical lines separating the regions with $m=0$ and $m \neq 0$, the full (dotted) lines represent continuous (first-order) phase transitions and the black dots denote tricritical points; for the values of $H_{0} / J$ chosen, one has: (a) two tricritical points at finite temperatures; (b) the lower tricritical point at zero temperature, defining the corresponding threshold value $H_{0}^{(2)}(\sigma)$.

left end limit of such a region; as a consequence of this, the lines AT1 and AT2 do not meet at the corresponding Independent-Ferromagnetic critical frontier.

Additional phase diagrams are shown in Fig. 4, where we exhibit two typical cases for the random-field width $(\sigma / J)=0.6$. In Fig. 4(a) we show the equivalent of Fig. 3(b), where two tricritical points appear at finite temperatures; now one gets qualitatively a similar effect, but with a random-field distribution characterized by a smaller ratio $H_{0} / \sigma$. From the quantitative point of view, the following changes occur, in the critical frontier Independent-Ferromagnetic, due to an increase in $\sigma / J$ : (i) such a critical frontier moves to higher values of $J_{0} / J$, leading to an enlargement of the Independent phase [corresponding to the region occupied by the $\mathrm{P}$ and $\mathrm{SG}$ phases of Fig. 4(a)]; (ii) the two tricritical points are shifted to lower temperatures. In Fig. 4(b) we present the situation of a zerotemperature tricritical point, defining the corresponding threshold value $H_{0}^{(2)}(\sigma)$; once again, one gets a physical situation similar to the one exhibited in Fig. 3(c), but with a much smaller ratio $H_{0} / \sigma$. Qualitatively similar effects may be also observed for other values of $\sigma$, but with different threshold values, $H_{0}^{(1)}(\sigma)$ and $H_{0}^{(2)}(\sigma)$. We have noticed 


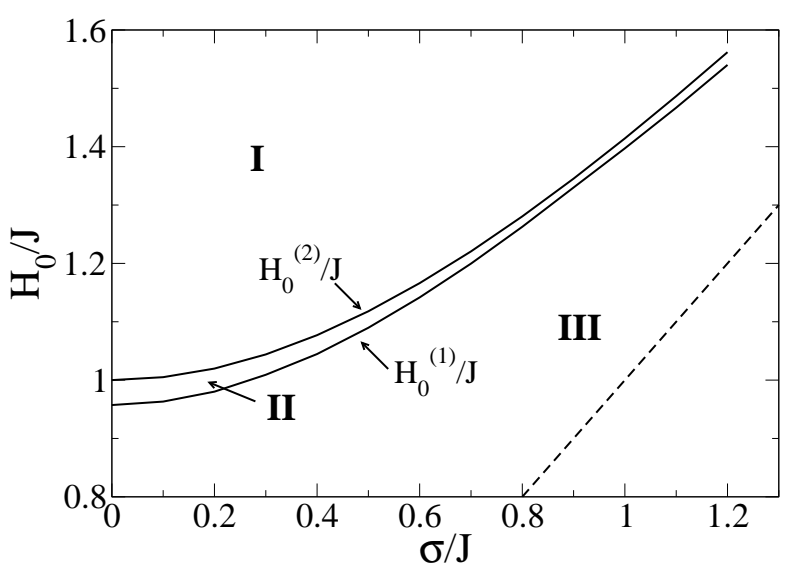

FIG. 5: Evolution of the threshold values $H_{0}^{(1)}(\sigma)$ (lower curve) and $H_{0}^{(2)}(\sigma)$ (upper curve) with the width $\sigma$ (all variables are scaled in units of $J$ ). Three distinct regions (I,II, and III) are shown, concerning the existence of tricritical points and first-order phase transitions along the Independent-Ferromagnetic critical frontier. The dashed straight line corresponds to $H_{0}=\sigma$, above which one has a tricritical point in the corresponding RFIM [30].

that such threshold values increase with $\sigma / J$, even though one requires less-pronounced double-peaked distributions [i.e., smaller values for the ratios $H_{0} / \sigma$ ] in such a way to get significant changes in the standard SK model phase diagrams [as can be seen in Figs. 2, $3(\mathrm{c})$, and $4(\mathrm{~b})]$.

The evolution of the threshold values $H_{0}^{(1)}(\sigma)$ and $H_{0}^{(2)}(\sigma)$ with the dimensionless width $\sigma / J$ is exhibited in Fig. 5. One notices three distinct regions in what concerns the existence of tricritical points and first-order phase transitions along the IndependentFerromagnetic critical frontier. Throughout region I [defined for $H_{0}>H_{0}^{(2)}(\sigma)$ ] a firstorder phase transition occurs at finite temperatures and reaches the zero-temperature axis; a single tricritical point is found at finite temperatures [a typical example is shown in Fig. 3(d)]. In region II [defined for $H_{0}^{(1)}(\sigma)<H_{0}<H_{0}^{(2)}(\sigma)$ ] one finds two finitetemperature tricritical points, with a first-order line between them [typical examples are exhibited in Figs. 3(b) and 4(a)]. Along region III $\left[H_{0}<H_{0}^{(1)}(\sigma)\right]$ one has a completely continuous Independent-Ferromagnetic critical frontier [like in Fig. 3(a)]. The dashed straight line corresponds to $H_{0}=\sigma$, which represents the threshold for the existence of a tricritical point in the corresponding RFIM [30]. Hence, if one associates the occurrence 


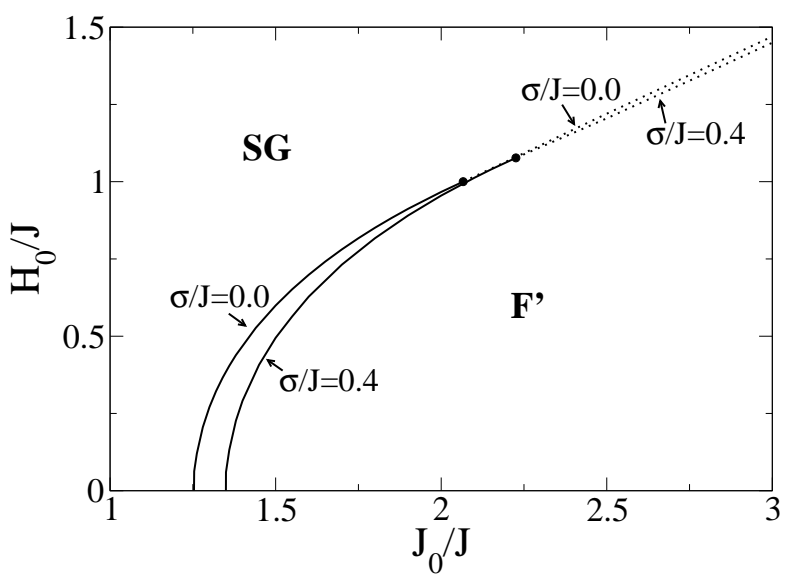

FIG. 6: The zero-temperature phase diagram $H_{0}$ versus $J_{0}$ (in units of $\mathrm{J}$ ) for two typical values of the dimensionless width $\sigma / J$. The critical frontiers separating the phases $\mathrm{SG}$ and $\mathrm{F}^{\prime}$ is continuous for small values of $H_{0} / J$ (full lines) and become first-order for higher values of $H_{0} / J$ (dotted lines); the black dots denote tricritical points. Although the two critical frontiers become very close near the tricritical points, they do not cross each other; the tricritical point located at a higher value of $J_{0} / J$ corresponds to the higher dimensionless width $\sigma / J$.

of tricritical points in the present model with those of the RFIM, one notices that such effects are attenuated due to the bond randomness, in agreement with Refs. [34, 35]; herein, the bond randomness introduces a spin-glass order parameter, in such a way that one needs stronger values of $H_{0} / J$ for these tricritical points to occur.

Let us now consider the phase diagram of the model at zero temperature; in this case, the spin-glass order parameter is trivial $(q=1)$, in such a way that the free energy and magnetization become,

$$
\begin{gathered}
f=-\frac{J_{0}}{2} m^{2}-\frac{H_{0}}{2}\left[\operatorname{erf}\left(\frac{J_{0} m+H_{0}}{J G_{0} \sqrt{2}}\right)-\operatorname{erf}\left(\frac{J_{0} m-H_{0}}{J G_{0} \sqrt{2}}\right)\right] \\
-\frac{J}{\sqrt{2 \pi}} G_{0}\left\{\exp \left[-\frac{\left(J_{0} m+H_{0}\right)^{2}}{2 J^{2} G_{0}^{2}}\right]+\exp \left[-\frac{\left(J_{0} m-H_{0}\right)^{2}}{2 J^{2} G_{0}^{2}}\right]\right\} \\
m=\frac{1}{2} \operatorname{erf}\left(\frac{J_{0} m+H_{0}}{J G_{0} \sqrt{2}}\right)+\frac{1}{2} \operatorname{erf}\left(\frac{J_{0} m-H_{0}}{J G_{0} \sqrt{2}}\right)
\end{gathered}
$$


where

$$
G_{0}=\left[1+\left(\frac{\sigma}{J}\right)^{2}\right]^{1 / 2}
$$

Using a procedure similar to the one applied for finite temperatures, one may expand Eq. (29) in powers of $m$,

$$
m=a_{1} m+a_{3} m^{3}+a_{5} m^{5}+O\left(m^{7}\right)
$$

where

$$
\begin{aligned}
& a_{1}=\sqrt{\frac{2}{\pi}} \frac{1}{G_{0}}\left(\frac{J_{0}}{J}\right) \exp \left(-\frac{H_{0}^{2}}{2 J^{2} G_{0}^{2}}\right), \\
& a_{3}=\frac{1}{6} \sqrt{\frac{2}{\pi}} \frac{1}{G_{0}^{3}}\left(\frac{J_{0}}{J}\right)^{3}\left\{\frac{1}{G_{0}^{2}}\left(\frac{H_{0}}{J}\right)^{2}-1\right\} \exp \left(-\frac{H_{0}^{2}}{2 J^{2} G_{0}^{2}}\right), \\
& a_{5}=\frac{1}{120} \sqrt{\frac{2}{\pi}} \frac{1}{G_{0}^{5}}\left(\frac{J_{0}}{J}\right)^{5}\left\{\frac{1}{G_{0}^{4}}\left(\frac{H_{0}}{J}\right)^{4}-\frac{6}{G_{0}^{2}}\left(\frac{H_{0}}{J}\right)^{2}+3\right\} \exp \left(-\frac{H_{0}^{2}}{2 J^{2} G_{0}^{2}}\right) .
\end{aligned}
$$

For $\left[H_{0} /\left(J G_{0}\right)\right]^{2}<1$ [i.e., $\left.a_{3}<0\right]$, we have a continuous critical frontier given by $a_{1}=1$,

$$
\frac{J_{0}}{J}=\sqrt{\frac{\pi}{2}} G_{0} \exp \left[\frac{H_{0}^{2}}{2 J^{2} G_{0}^{2}}\right]
$$

This continuous critical frontier ends at a tricritical point $\left(a_{3}=0\right)$,

$$
\frac{1}{G_{0}^{2}}\left(\frac{H_{0}}{J}\right)^{2}=1 \Rightarrow \frac{H_{0}}{J} \equiv \frac{H_{0}^{(2)}}{J}=\left[1+\left(\frac{\sigma}{J}\right)^{2}\right]^{1 / 2},
$$

which may be substituted in Eq. (35) to give

$$
\frac{J_{0}}{J}=\sqrt{\frac{\pi e}{2}}\left[1+\left(\frac{\sigma}{J}\right)^{2}\right]^{1 / 2}
$$



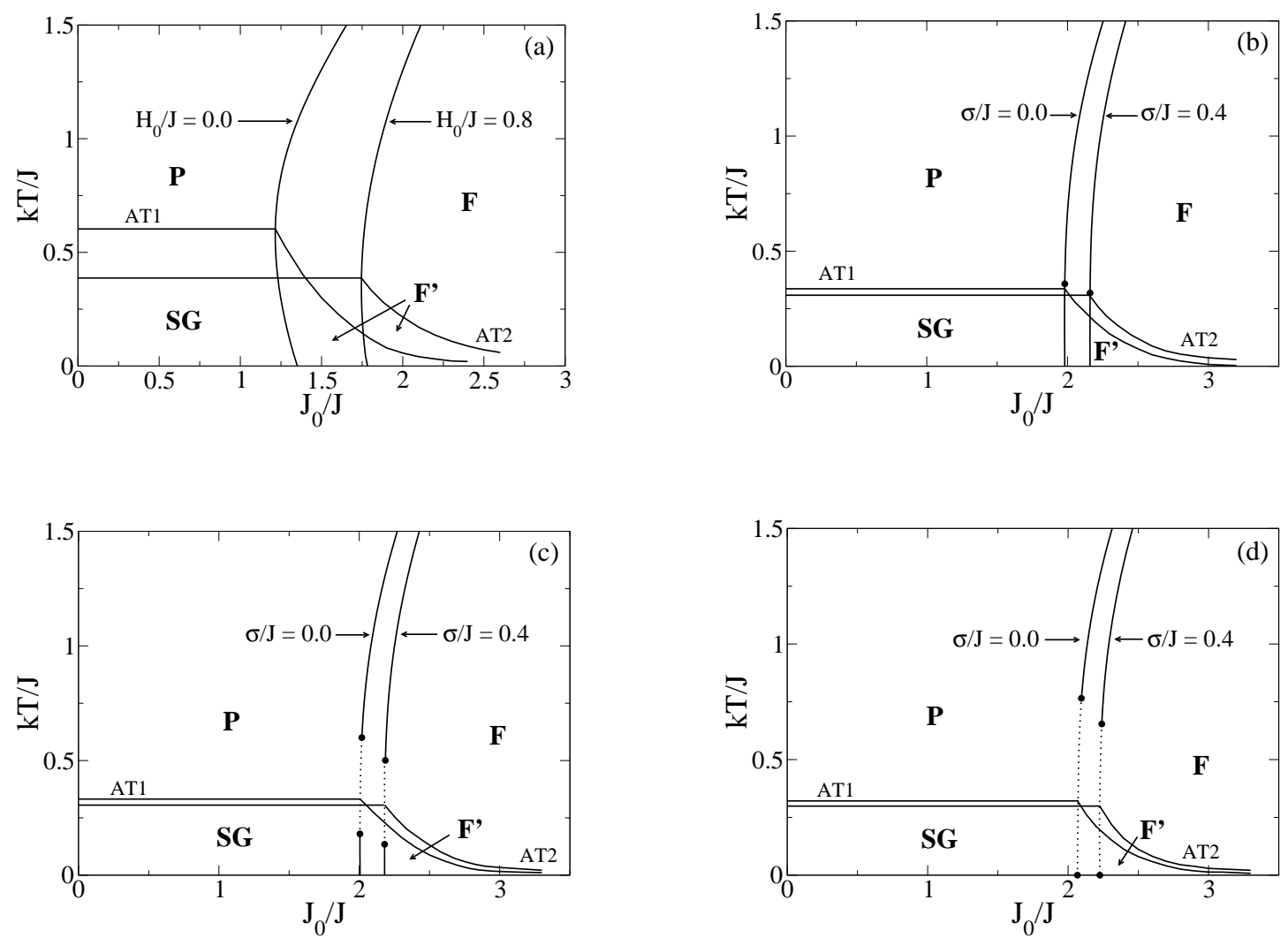

FIG. 7: Typical phase diagrams of the infinite-range-interaction ISG in the presence of a doubleGaussian random field with $(\sigma / J)=0.4$ are compared with those already known for some particular cases. Comparisons of qualitatively similar phase diagrams are presented, essentially in what concerns the critical frontier that separates the regions with $m=0$ and $m \neq 0$. (a) Phase diagrams for the single Gaussian $\left[\left(H_{0} / J\right)=0.0\right.$ and $\left.(\sigma / J)=0.4\right]$ and the double Gaussian $\left[\left(H_{0} / J\right)=0.8\right.$ and $\left.(\sigma / J)=0.4\right]$ distributions for the random fields. (b) Phase diagrams for the bimodal $\left[\left(H_{0} / J\right)=0.9573\right]$ and the double Gaussian $\left[\left(H_{0} / J\right)=1.0447\right]$ distributions for the random fields. (c) Phase diagrams for the bimodal $\left[\left(H_{0} / J\right)=0.97\right]$ and the double Gaussian $\left[\left(H_{0} / J\right)=1.055\right]$ distributions for the random fields. (d) Phase diagrams for the bimodal $\left[\left(H_{0} / J\right)=1.0\right]$ and the double Gaussian $\left[\left(H_{0} / J\right)=1.077\right]$ distributions for the random fields. The phases are labelled according to the definitions in the text. AT1 and AT2 denote AT lines, and all variables are scaled in units of $J$. 

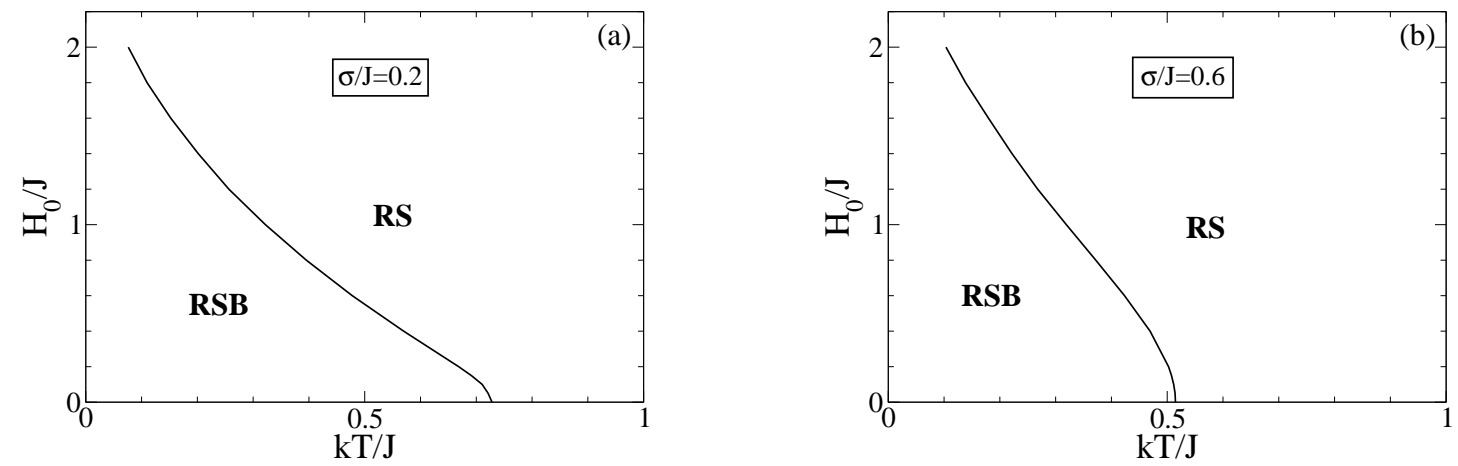

FIG. 8: Instabilities of the replica-symmetric solution of the infinite-range-interaction ISG (cases $J_{0}=0$ ) in the presence of a double-Gaussian random field, for two typical values of distribution widths: (a) $(\sigma / J)=0.2 ;$ (b) $(\sigma / J)=0.6$. In each case the AT line separates a region of RS from the one characterized by RSB (all variables are scaled in units of $J$ ).

Hence, Eqs. (36) and (37) yield the coordinates of the tricritical point at zero temperature. In addition to that, the result of Eq. (36) corresponds to the exact threshold value $H_{0}^{(2)}(\sigma)$ (as exhibited in Fig. 5). The above results are represented in the zero-temperature phase diagram shown in Fig. 6, where one finds a single critical frontier separating the phases $\mathrm{SG}$ and $\mathrm{F}^{\prime}$.

In order to illustrate that the present model is capable of reproducing qualitatively the phase diagrams of previous works, namely, the Ising spin glass in the presence of random fields following either a Gaussian [24], or a bimodal [25] probability distribution, in Fig. 7 we compare typical results obtained for the Ising spin-glass model in the presence of a double Gaussian distribution characterized by $(\sigma / J)=0.4$ with those already known for such particular cases. In these comparisons, we have chosen qualitatively similar phase diagrams, mainly taking into account the critical frontier that separates the regions with $m=0$ and $m \neq 0$. In Fig. $7($ a) we exhibit the phase diagram of the present model $\left[\left(H_{0} / J\right)=0.8\right]$ together with the one of an ISG in the presence of random fields described by a single Gaussian distribution; both phase diagrams are qualitatively similar to the one of the standard SK model. In Fig. 7(b) we present phase diagrams for the bimodal and double Gaussian distributions, at their corresponding threshold values, $H_{0}^{(1)}(\sigma)$. Typical situations for the cases of the bimodal and double Gaussian distributions, where two tricritical points appear along the critical frontier that separates the regions with $m=0$ 
and $m \neq 0$, are shown in Fig. 7(c). Phase diagrams for the bimodal and double Gaussian distributions, at their corresponding threshold values, $H_{0}^{(2)}(\sigma)$, are presented in Fig. $7(\mathrm{~d})$.

Next, we analyze the AT instability for $J_{0}=0$; in this case, Eq. (16) may be written as

$$
\left(\frac{k T}{J}\right)^{2}=\frac{1}{\sqrt{2 \pi}} \int_{-\infty}^{+\infty} d z e^{-z^{2} / 2} \operatorname{sech}^{4} \beta J\left\{G z+\frac{H_{0}}{J}\right\}
$$

which corresponds to the same instability found in the case of a single-Gaussian random field [24]. In Fig. 8 we exhibit AT lines for two typical values of distribution widths; in each case the AT line separates a region of RS from the one characterized by RSB. One notices that the region associated with RSB gets reduced for increasing values of $\sigma$; however, the most interesting aspect in these lines corresponds to an inflection point, which may be identified with the one that has been observed in the experimental equilibrium boundary of the compound $\mathrm{Fe}_{\mathrm{x}} \mathrm{Zn}_{1-\mathrm{x}} \mathrm{F}_{2}$ [15, 24]. Up to now, this effect was believed to be explained only through the ISG in the presence of a single-Gaussian random field, for which the phase diagrams in the cases $J_{0}>0$ are much simpler, with all phase transitions being continuous, typically like those of the SK model. Herein, we have shown that an inflection point in the AT line may also occur in the present model, for which one has a wide variety of phase diagrams in the corresponding case $J_{0}>0$, as exhibited above. Therefore, the present model would be appropriate for explaining a similar effect that may be also observed experimentally in diluted antiferromagnets characterized by first-order phase transitions, like $\mathrm{Fe}_{\mathrm{x}} \mathrm{Mg}_{1-\mathrm{x}} \mathrm{Cl}_{2}$.

\section{CONCLUSIONS}

We have studied an Ising spin-glass model, in the limit of infinite-range interactions and in the presence of random magnetic fields distributed according to a double-Gaussian probability distribution. Such a distribution contains, as particular limits, both the singleGaussian and bimodal probability distributions. By varying the parameters of this distri- 
bution, a rich variety of phase diagrams is obtained, with continuous and first-order phase transitions, as well as tricritical points. The condition for the existence of a single finitetemperature tricritical point at the critical frontier Paramagnetic-Ferromagnetic (i.e., in the region of stability of the replica-symmetric solution), characterized by a first-order line at low temperatures, is derived analytically. Besides that, we found an inflection point in the AT line (in the plane magnetic field versus temperature), which may correspond to the one observed in the compound $\mathrm{Fe}_{\mathrm{x}} \mathrm{Zn}_{1-\mathrm{x}} \mathrm{F}_{2}[15,24]$. This effect, which has already shown up in the Ising spin-glass model in the presence of a Gaussian random field [24], is herein obtained for a more general probability distribution for the magnetic fields. Hence, the present model is appropriate for explaining a similar effect that could be observed also in diluted antiferromagnets characterized by first-order phase transitions, like $\mathrm{Fe}_{\mathrm{x}} \mathrm{Mg}_{1-\mathrm{x}} \mathrm{Cl}_{2}$. Therefore, with this random-field distribution, one may adjust the model to given physical situations, in order to reproduce a wide diversity of effects that occur in real systems.

The double-Gaussian probability distribution, defined above, is suitable for a theoretical description of random-field systems, being a better candidate for such a purpose than the two most commonly used distributions in the literature, namely, the bimodal and single-Gaussian distributions, due to the following reasons: (i) In the identifications of the RFIM with diluted antiferromagnets in the presence of a uniform magnetic field, the local random fields are expressed in terms of quantities that vary in both sign and magnitude [28, 29]. This characteristic rules out the bimodal probability distribution from such a class of physical systems. The double-Gaussian probability distribution is appropriate for a description of diluted antiferromagnets for a large range of magnetic concentrations, like in the RFIM, as well as in the ISG regimes. (ii) Although the RFIM defined in terms of a single-Gaussian probability distribution for the fields is physically acceptable, it usually leads to a continuous phase transition at finite temperatures, either within mean-field [31, 32, 33], or standard short-range-interaction approaches [36, 37]. Such a system is not able to exhibit first-order phase transitions and tricritical points, that may occur in some diluted antiferromagnets [14]. A similar behavior was obtained for an ISG in the presence of random magnetic fields following a single-Gaussian probability distribution, where all phase transitions were found to be continuous [24]. The present model, defined in terms of a double-Gaussian probability distribution, is expected to be relevant for $\mathrm{Fe}_{\mathrm{x}} \mathrm{Mg}_{1-\mathrm{x}} \mathrm{Cl}_{2}$ 
(which is known to exhibit a first-order phase transition in the RFIM regime [14]) with concentrations $x<0.55$, where the ISG behavior shows up.

\section{Acknowledgments}

We thank Prof. E. M. F. Curado for fruitful conversations. The partial financial supports from CNPq and Pronex/MCT/FAPERJ (Brazilian agencies) are acknowledged.

[1] V. Dotsenko, Introduction to the Replica Theory of Disordered Statistical Systems (Cambridge University Press, Cambridge, 2001).

[2] H. Nishimori, Statistical Physics of Spin Glasses and Information Processing (Oxford University Press, Oxford 2001).

[3] K. H. Fischer and J. A. Hertz, Spin Glasses (Cambridge University Press, Cambridge, 1991).

[4] K. Binder and A. P. Young, Rev. Mod. Phys. 58, 801 (1986).

[5] Spin Glasses and Random Fields, edited by A. P. Young (World Scientific, Singapore, 1998).

[6] D. Sherrington and S. Kirkpatrick, Phys. Rev. Lett. 35, 1792 (1975).

[7] G. Parisi, Phys. Rev. Lett. 43 , 1754 (1979); 50, 1946 (1983).

[8] J. R. L. de Almeida and D. J. Thouless, J. Phys. A 11, 983 (1978).

[9] A. P. Young and H. G. Katzgraber, Phys. Rev. Lett. 93, 207203 (2004).

[10] H. G. Katzgraber and A. P. Young, Phys. Rev. B 72, 184416 (2005).

[11] D. S. Fisher and D. A. Huse, Phys. Rev. Lett. 56, 1601 (1986).

[12] W. L. McMillan, J. Phys. C 17 , 3179 (1984).

[13] A. J. Bray and M. A. Moore, in Heidelberg Colloquium on Glassy Dynamics, Lecture Notes in Physics 275 , edited by J. L. van Hemmen and I. Morgenstern (Springer-Verlag, Heidelberg 1987). 
[14] D. P. Belanger, in Spin Glasses and Random Fields, edited by A. P. Young (World Scientific, Singapore, 1998).

[15] F. C. Montenegro, A. R. King, V. Jaccarino, S-J. Han and D. P. Belanger, Phys. Rev. B 44,2155 (1991).

[16] D. P. Belanger, Wm. E. Murray Jr., F. C. Montenegro, A. R. King, V. Jaccarino and R. W. Erwin, Phys. Rev. B 44, 2161 (1991).

[17] A. Rosales-Rivera, J. M. Ferreira, and F. C. Montenegro, Europhys. Lett. 50, 264 (2000).

[18] J. Kushauer and W. Kleemann, J. Magn. Magn. Mater. 140-144, 1551 (1995).

[19] J. Kushauer, R. van Bentum, W. Kleemann, and D. Bertrand, Phys. Rev. B 53, 11647 (1996).

[20] W. C. Barber and D. P. Belanger, J. Magn. Magn. Mater. 226-230 , 545 (2001).

[21] P. H. R. Barbosa, E. P. Raposo, and M. D. Coutinho, J. Magn. Magn. Mater. 226-230 , 1293 (2001).

[22] P. H. R. Barbosa, E. P. Raposo, and M. D. Coutinho, Phys. Rev. Lett. 91 , 197207 (2003).

[23] P. H. R. Barbosa, E. P. Raposo, and M. D. Coutinho, Phys. Rev. B 72, 092401 (2005).

[24] R. F. Soares, F. D. Nobre and J. R. L. de Almeida, Phys. Rev. B 50 , 6151 (1994).

[25] E. Nogueira, Jr., F. D. Nobre, F. A. da Costa, and S. Coutinho, Phys. Rev. E 57 , 5079 (1998); Erratum, Phys. Rev. E 60 , 2429 (1999).

[26] J. M. de Araújo, F. D. Nobre, and F. A. da Costa, Phys. Rev. E 61, 2232 (2000).

[27] S. R. Vieira, F. D. Nobre, and C. S. O. Yokoi, Phys. Rev. E 61, 4760 (2000).

[28] S. Fishman and A. Aharony, J. Phys. C 12 , L729 (1979).

[29] J. Cardy, Phys. Rev. B 29 , 505 (1984).

[30] N. Crokidakis and F. D. Nobre, J. Phys. Condens. Matter 20, 145211 (2008).

[31] A. Aharony, Phys. Rev. B 18, 3318 (1978).

[32] D. Andelman, Phys. Rev. B 27, 3079 (1983).

[33] S. Galam and J. Birman, Phys. Rev. B 28, 5322 (1983).

[34] M. Aizenman and J. Wehr, Phys. Rev. Lett. 62, 2503 (1989); Erratum, Phys. Rev. Lett. 64, 1311 (1990).

[35] K. Hui and A.N. Berker, Phys. Rev. Lett. 62, 2507 (1989); Erratum, Phys. Rev. Lett. 63, 2433 (1989). 
[36] M. Gofman, J. Adler, A. Aharony, A. B. Harris and M. Schwartz, Phys. Rev. B 53, 6362 (1996).

[37] M. R. Swift, A. J. Bray, A. Maritan, M. Cieplak and J. R. Banavar, Europhys. Lett. 38, 273 (1997). 